\title{
Flow field assessment in a vertical axis wind turbine
}

\author{
R. Ricci, S. Montelpare, A. Secchiaroli \& V. D’Alessandro \\ Dipartimento di Energetica, Università Politecnica delle Marche, Italy
}

\begin{abstract}
In this work, flow field features in a vertical axis Savonius wind rotor were investigated by means of a specially developed CFD method validated using wind tunnel testing data.

The Savonius rotor exhibits a strong variation of its angular velocity during the rotation. The solution of the second cardinal equation of dynamics is then needed to obtain a high-fidelity physical modelling of the device and an accurate performance prediction.

The proposed methodology is based on the integration between a commercial CFD code and custom MatLab routines. The former is used for the flow field computations, while the latter are used for the time integration of the second cardinal equation of dynamics. Unsteady RANS equations closed with the v2-f turbulence model were solved to consider the turbulent flow behaviour.

This procedure allows one to numerically evaluate the dimensionless parameters used in the performance characterization of wind turbines. Moreover, the flow field around the rotor can be evaluated, at every solved angular position (with respect to the incoming wind direction), taking into account the effect of the angular velocity variations of the rotor. An extended wind tunnel testing campaign was conducted on a rotor model in order to validate the computational procedure. A very good agreement between experimental and numerical data can be observed.

The main features of the flow field at different operating points of the rotor are presented in this paper. An evaluation of the characteristic of the wake behind the rotor obtained by means of a spectral analysis of the velocity magnitude signal is reported too.
\end{abstract}

Keywords: computational fluid-dynamics, Savonius wind rotor, wind energy. 


\section{Introduction}

Nowadays, vertical axis wind turbines are under the study of several research groups because they are very attractive for the integration of wind turbines in urban environments.

Two main types of vertical axis wind turbines exist: Darrieus rotor and Savonius rotor. In the first case, the lift force is responsible for the motion whereas in the second case the drag force is the main force which makes the turbine to spin.

In this study, a detailed numerical analysis of the flow field developing around a Savonius wind rotor is presented in order to gain insight into the basic mechanisms on which torque release is based.

Previous numerical studies about Savonius rotor aerodynamic performance are available in literature [5-10]. Numerical computations which take into account the rotor rotation following two different approaches: locating the rotor in a noninertial frame or in an inertial one. In the first case, the solution obtained is strictly connected with the angle of attack of the rotor (angle between the cord of the rotor and the wind direction). Moreover, Coriolis force effect is completely neglected in Eddy-Viscosity Models (EVM) using the transport equation for TKE. This is directly related to the scalar nature of the TKE [3].

Locating the rotor in a inertial reference frame with constant angular velocity a not high-fidelity physical modelling is performed because the Savonius during its rotation exhibits a strong variation in angular velocity due to its torque release; for this reason in this work an approach based on an inertial frame with variable rotor angular velocity was used. RANS equations (closed using $\overline{v^{2}}-f$ turbulence model) were solved in order to obtain accurate information about the flow field. The rotor blades were treated as rigid bodies and their behaviour was modelled by means of the Second Cardinal Equation of Dynamics.

An extended wind tunnel testing program was also conducted at the Environmental Wind Tunnel of the Polytechnic University of Marche [4]. The experimental facilities allowed to evaluate rotor performance expressed by means of the dimensionless parameters usually used in the aerodynamics of wind turbines [1]. The experimental data were used to validate the developed computational methodology.

\section{Mathematical model}

The Savonius rotor analyzed in this work is a split-type as clearly shown in Fig. 1 and it has the characteristic angle of the helix fixed to zero. This allows one to build a computational $2 \mathrm{D}$ domain named $\Omega$.

A crucial issue in the analysis of the flow field around the Savonius rotor is the treatment of the fluid-solid coupling and its modelling. In this work the structure (rotor) was treated as a rigid body while the fluid was modelled as incompressible and viscous. 


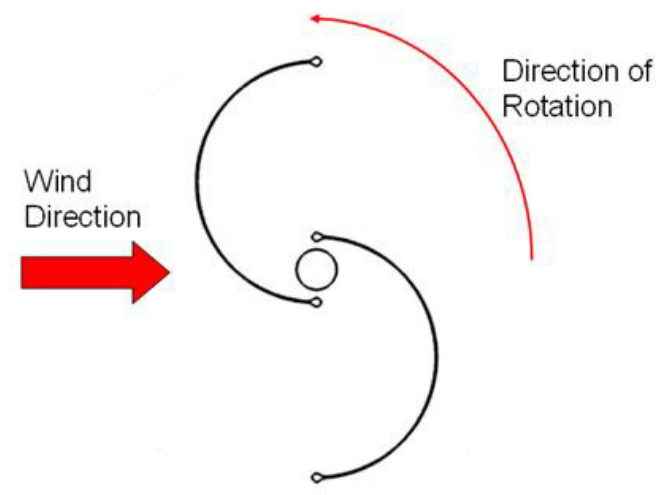

Figure 1: Savonius rotor analyzed in this work.

The fluid-structure coupling algorithm presented in this paper provides, for each time-step, the solution of the fluid-dynamic field (by means of the finite volume code FLUENT) and then evaluates the angular velocity of the rotating system. These analyses were performed by a numerical algorithm written in MatLab language. A flow chart of the algorithm is shown in Fig. 2.

The fluid flow induces the motion of the solid body by means of aerodynamic interactions. Only the rigid body kinematics was considered; hence the motion of the solid is evaluated integrating the second cardinal equation of dynamics for 1DOF (Degree of Freedom) rotating system (1)

$$
I \dot{\omega}=\sum_{k} M_{G, k}^{e x t}
$$

which is referred to the fixed rotation axis of the system. Torques acting on the rotor are the aerodynamic moment $\mathrm{M}_{\mathrm{a}}$ and resistant torque $\mathrm{M}_{\mathrm{r}}$. The first is expressed per unit of rotor length by (2), in which $n_{k}$ are the components of the outward normal unit vector while the second one can be expressed as a linear function of the angular velocity in order to reproduce the experimental conditions described in [4].

$$
M_{a}^{\prime}=\int_{\partial \Omega_{s}} \varepsilon_{3 i j}\left(x_{i}-x_{G, i}\right) \tau_{j k} n_{k} d \Gamma
$$

with

$$
\begin{gathered}
\tau_{i j}=\left(\mu+\mu_{T}\right)\left(\partial_{i} u_{j}+\partial_{j} u_{i}\right)-p \delta_{i j} \\
M_{r}=a_{k} \omega
\end{gathered}
$$

Second cardinal equations of dynamics can be written as:

$$
I \dot{\omega}=L \mathrm{M}_{\mathrm{a}}^{\prime}-\mathrm{M}_{\mathrm{r}}
$$


where $\mathrm{L}$ is the rotor height. The initial condition adopted in solving (5) is given by:

$$
\omega(0)=\omega_{0}
$$

The rotor moment of inertia resulted from a CAD evaluation of the model geometry. $\omega_{0}$ was assumed to be $16 \mathrm{rad} / \mathrm{s}$ in order to achieve a suitable time-step size in the first time-step solved for flow governing equations numerical solution.

The strategy used in this work in order to solve the fluid-structure interaction problem was a SMM (Sliding Mesh Model) approach. Hence, in $\Omega$ an inner subdomain $\mathrm{K}$ is defined.

$\mathrm{K}$ represents a rotating frame around a fixed axis normal to the represented plane and passing for the point $\left(x_{G}, y_{G}\right)$ with radium $\mathrm{R}_{\mathrm{mg}}$ (red area represented in Fig. 2).

In other words, the computational grid is decomposed into two sub-grids, where the inner $\mathrm{K}$ domain is able to rigidly rotate with respect to the outer one.

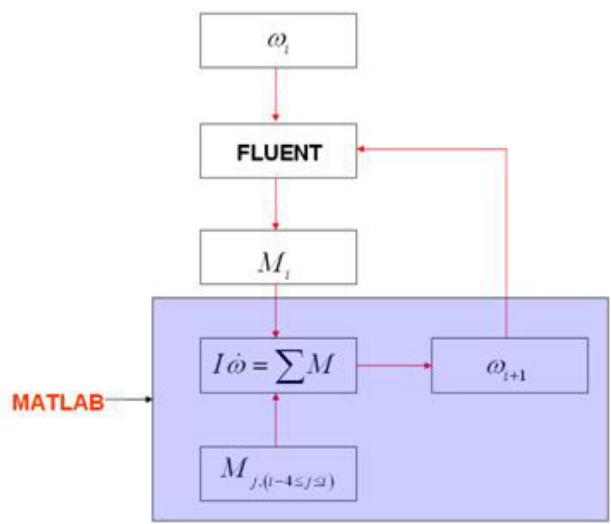

Figure 2: $\quad$ Flow chart of the computational methodology.

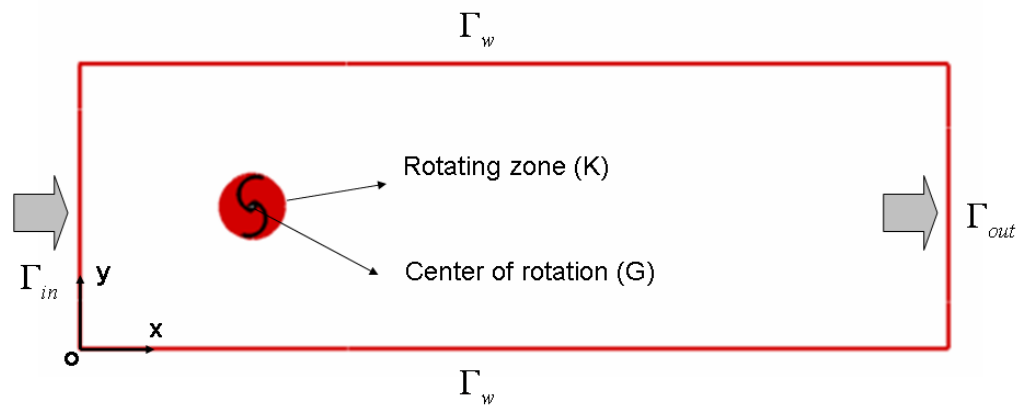

Figure 3: $\quad$ Sketch of the computational model. 
The commonly used Fluid-Dynamics conservation laws (i.e. momentum, mass and turbulence quantities) must be suitably modified in order to take into account the grid motion. At this purpose a mesh motion flux term, related to mesh motion velocity $\mathbf{u}_{\mathbf{g}}$, was introduced. Hence, the transport equation for a generic fluid flow variable $\phi$ becomes:

$$
\int_{\Omega} \partial_{t}(\rho \phi) d \Omega+\oint_{\partial \Omega} \rho\left(\mathbf{u}-\mathbf{u}_{\mathbf{g}}\right) \phi \cdot \mathbf{n} d \Gamma-\oint_{\partial \Omega}(\Gamma \nabla \phi) \cdot \mathbf{n} d \Gamma+\int_{\Omega} s d \Omega=0
$$

the main problem due to the relative motion between the mesh elements of the $\mathrm{K}$ and $\{\Omega \backslash \mathrm{K}\}$ domains is related to the fluxes at the boundaries. The adopted solution procedure provides an appropriate choice of time-step size (8) obtained by fixing the number of nodes that slide in each time-step and knowing the distance between two consecutive nodes on the grids interface $\Delta \mathrm{s}$, the angular velocity at the previous time-step solved $\omega^{(\mathrm{n}-1)}$ and the radius of the rotating frame $\mathrm{R}_{\mathrm{mg}}$. In this way there are not overlapped cells at the sliding boundary and a conformal grid is assured in every time-step solved.

$$
\Delta t^{(n)}=\frac{\Delta s \cdot N}{\omega^{(n-1)} \cdot R_{m g}}
$$

Moreover, regarding the meshing strategy, inside the $\mathrm{K}$ domain an unstructured triangular mesh having a curvilinear orthogonal refinement near the walls (Fig. 3) was adopted, in $\left\{\Omega_{\mathrm{F}} \backslash \mathrm{K}\right\}$ (the white area inside the rectangle) both curvilinear and orthogonal structured elements were used.

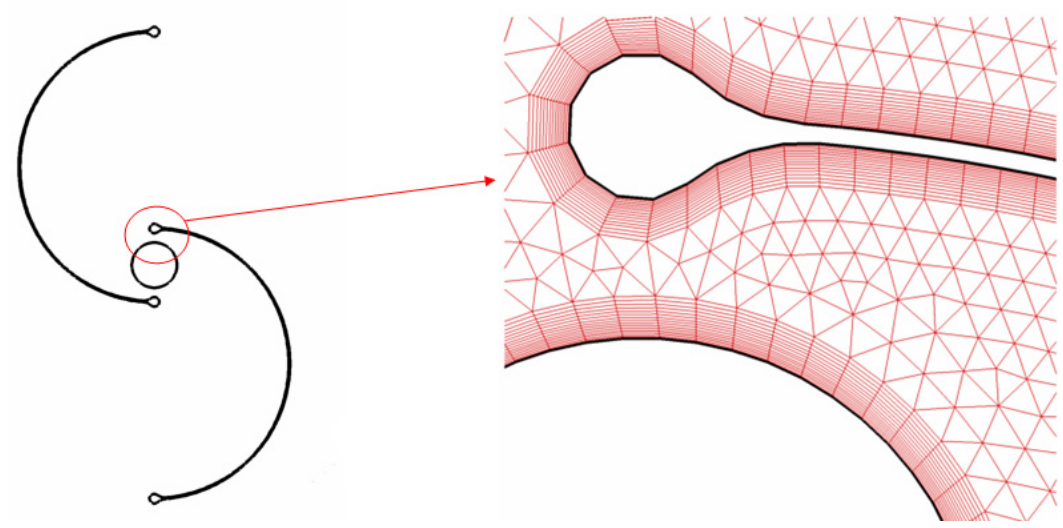

Figure 4: $\quad$ Meshing strategy used near the rotor blades. 
RANS equations closed with $\overline{v^{2}}-f$ model must be, obviously, completed with a set of boundary conditions. The imposed boundary conditions are:

- a fixed inflow velocity at the inflow section (see Fig. 2);

- a zero normal pressure gradient at the inflow section;

- $\quad$ no-slip at the rotor blades (9);

$$
\mathbf{u}(\mathbf{x})=\omega \mathbf{e}_{\mathbf{k}} \wedge\left(\mathbf{x}-\mathbf{x}_{\mathbf{G}}\right) \forall \mathbf{x} \in \partial \Omega_{s}
$$

- $\quad \Gamma_{\mathrm{w}}$ is treated as wall in order to reproduce the test chamber of the Environmental Wind Tunnel Laboratory of the Polytechnic University of Marche and to create a virtual wind tunnel.

- $\quad$ an outflow physical boundary condition was used at $\Gamma_{\text {out }}[11]$.

- the turbulent intensity and the hydraulic diameter were fixed on $\Gamma_{\text {in }}$ (the same values used in the experimental tests were reproduced).

- At $\Gamma_{\mathrm{in}}, \Gamma_{\mathrm{w}}$ and on the rotor blades a zero normal gradient condition for the turbulent quantities was fixed

- For the fluid domain the following initial condition was set:

$$
\mathbf{u}(\mathbf{x}, t=0)=\mathbf{0} \quad \forall \mathbf{x} \in \Omega \backslash K
$$

\section{Numerical methods}

RANS equations coupled with the turbulence model, were solved by means of a second order Finite Volume Method [13]. An extended dissertation about the numerical schemes used in the solution of the equations can be found in [2] and [3]. The fluid-structure coupling algorithm, presented in this paper, implies that the aerodynamic torque is known only from previous time-step. This requires an explicit scheme for the time-integration of the second cardinal equation of dynamics with a time-step size fixed for mesh conformity reasons, see (9). The code able to manage the fluid-structure interaction in this moment provides an explicit four stage Runge-Kutta method, a two point and a three point explicit Adams-Bashfort schemes. It was showed as an increasing in the scheme order produce a better agreement between and experimental data [3].

\section{Results}

Savonius wind rotor performance, obtained from numerical computations, was evaluated for every angular position occupied by the rotor using the parameters usually used in Aerodynamics of wind turbines [1]:

$$
C_{m}=\frac{M}{\frac{1}{2} \rho u_{\infty}^{2} A \cdot R}
$$




$$
\begin{gathered}
C_{P}=\frac{P}{\frac{1}{2} \rho u_{\infty}^{3} A} \\
\lambda=\frac{\omega R}{u_{\infty}}
\end{gathered}
$$

where $\mathrm{R}$ is the rotor radius and $\mathrm{A}$ is the rotor frontal area. The rotor performance is evaluated averaging on the single rotor rotation (12) and (13).

$$
\begin{aligned}
\langle\lambda\rangle & =\frac{1}{2 \pi} \int_{0}^{2 \pi} \lambda(\theta) d \theta \\
\left\langle C_{P}\right\rangle & =\frac{1}{2 \pi} \int_{0}^{2 \pi} C_{P}(\theta) d \theta
\end{aligned}
$$

The integrals (14) and (15) were calculated using a second order accurate method [3]. In Fig. 4 the effect of the average on the instantaneous tip speed ratio is shown. In Fig. 5 the mean power coefficient trend is reported.

The Savonius rotor mean performance, at the operating point, was evaluated averaging the parameters reported in Figs. 4 and 5 once the asymptotic behaviour was reached. The average was performed on the samples number corresponding to the steady condition. These data were used for numerical/experimental data comparison as reported in Fig. 6 and Fig. 7.

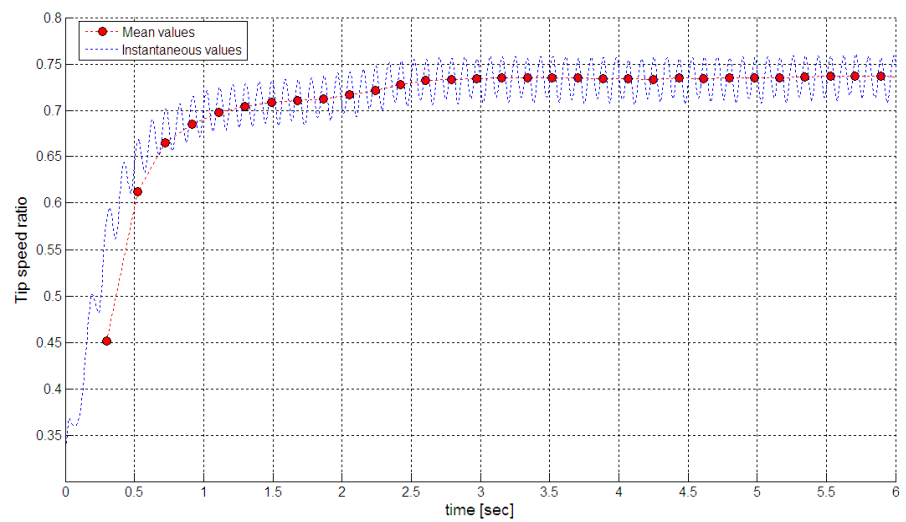

Figure 5: Instantaneous and averaged tip-speed ratio. 


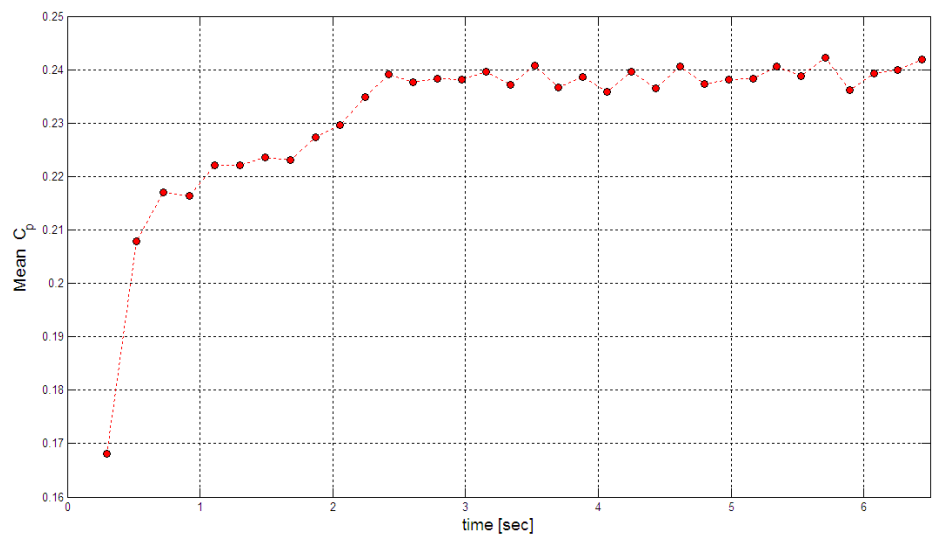

Figure 6: Mean power coefficient.

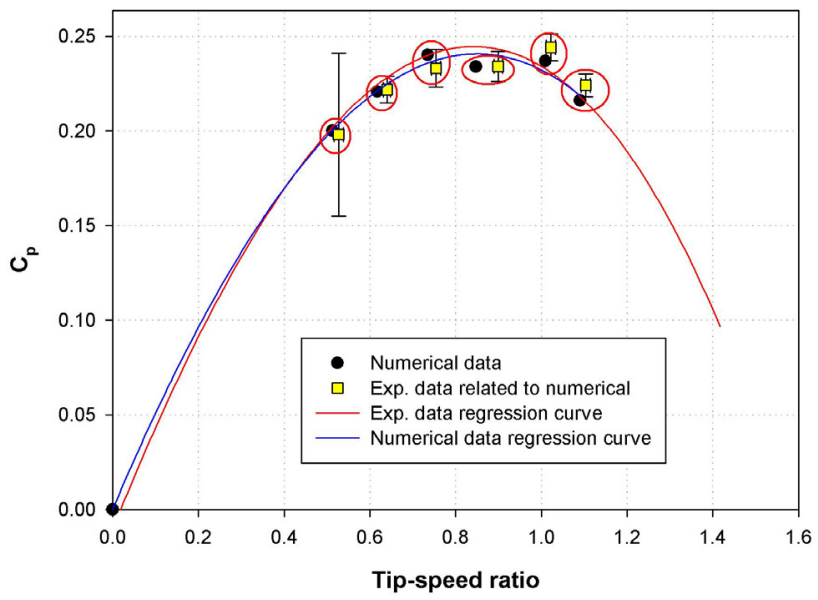

Figure 7: Comparison between numerical and experimental power.

Numerical and experimental data are reported in Fig. 6 and Fig. 7. Aerodynamic performance, obtained by the numerical simulations, shows a very good agreement with experimental data. This allows one to use the computational model for a fluid dynamic analysis of a Savonius wind rotor.

In all the simulations reported in this paper, the inflow wind velocity has been fixed to $9 \mathrm{~m} / \mathrm{sec}$ and the turbulent intensity at the inflow section was fixed to $0.2 \%$ as in the experimental set-up.

In order to gain an insight into the main feature of the flow field developing around the Savonius rotor several contours were produced and reported in 
Figs. 8, 9 and 10. Data related to the rotor with and AOA of about 45 degrees respect the incoming wind velocities were reported. TSRs reported are 0.735 , 0.847 and 1.1.This angular position was identified as the position of the rotor maximum torque release [3].

An increasing of the stagnation region on the returning blade can be observed going beyond the unitary tip-speed ratio. The stagnation region on the pressure side of the advancing undergoes a shift near the blade tip with the increase of the TSR. On the suction side of the advancing blade, the increase of the TSR produces a larger size of the depression zone. This feature causes a more important contribution of the lift force on the rotor torque release. A separation bubble is located on the suction side of the advancing blade; its extension seems to be independent by the TSR. The wake zone behind the returning blade is clearly more affected by the TSR values. The increase of TSR produces the born of complex structures behind the returning blade.

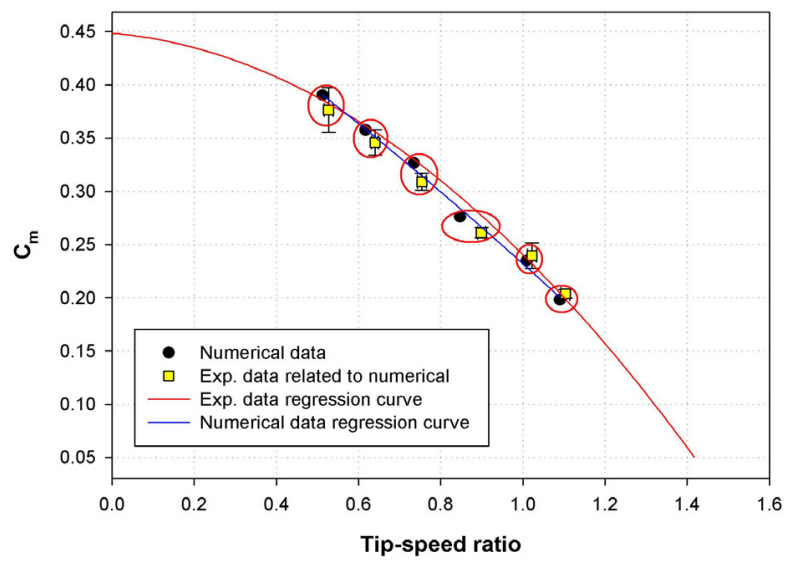

Figure 8: Comparison between numerical and experimental torque curve.

A crucial issue regarding the structural of design of this object is related to the vortex shedding frequencies. A PSD analysis of the absolute velocity magnitude signal was conducted on a grid of point reported in Fig 11. In the point located at (3D, -D) energy peak an in correspondence to the rotation frequency can be observed. This feature was found for point $(3 \mathrm{D}, \mathrm{D})$ too. In this case the energy peak related to the blade passage frequency is less pronounced. Moreover, this frequency corresponds with the vortex shedding frequency. 

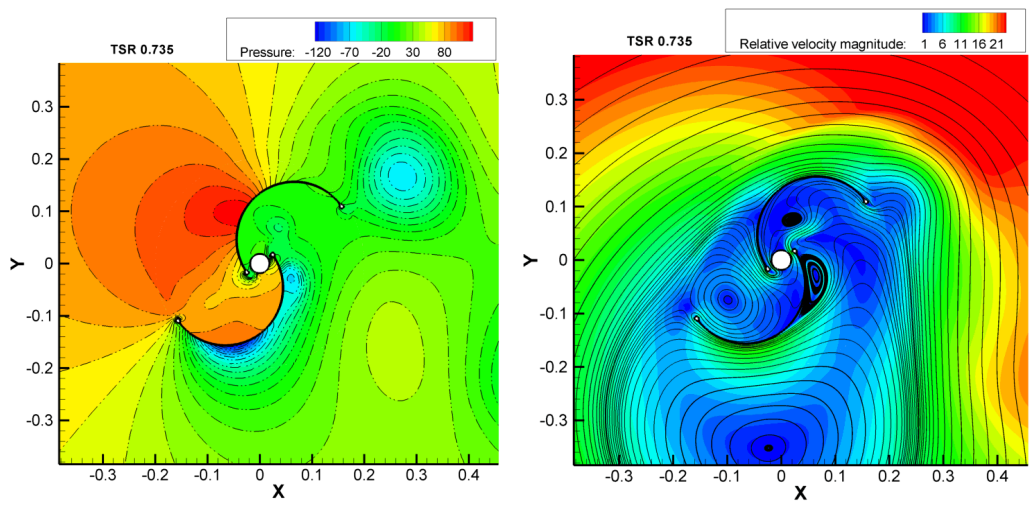

Figure 9: Contour of relative pressure and relative velocity magnitude. $\mathrm{TSR}=0.735$.
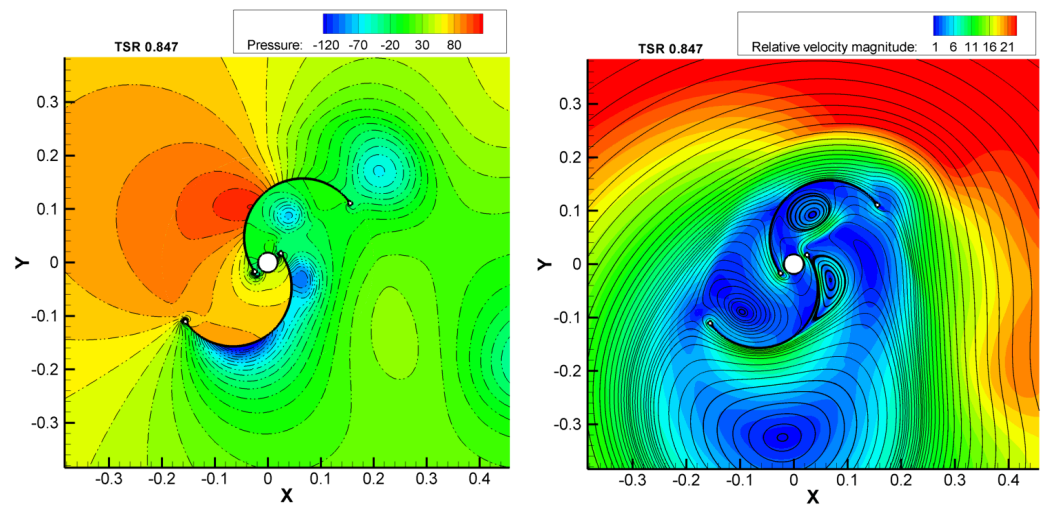

Figure 10: Contour of relative pressure and relative velocity magnitude. $\mathrm{TSR}=0.847$.
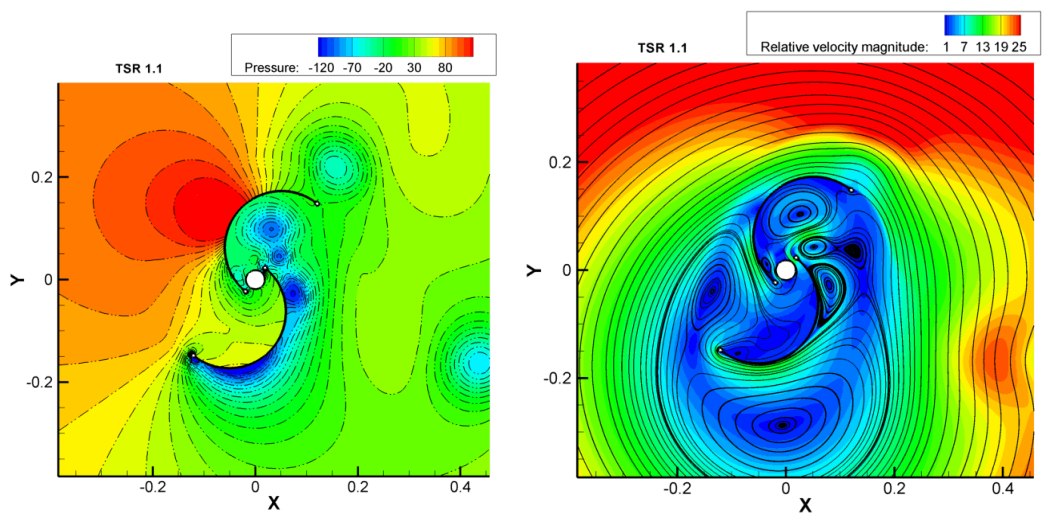

Figure 11: Contour of relative pressure and relative velocity magnitude. $\mathrm{TSR}=1.1$. 


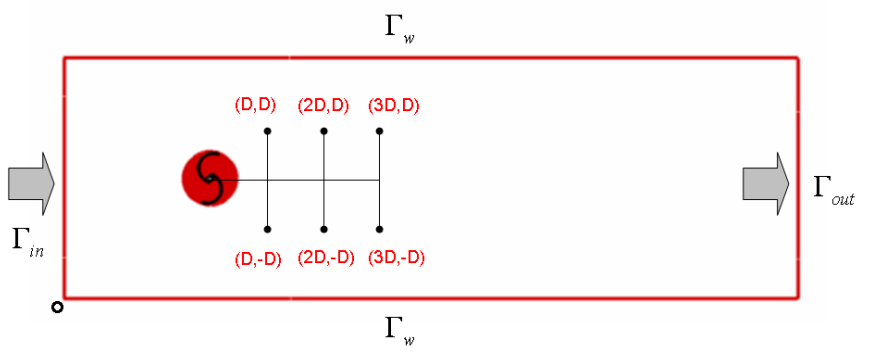

Figure 12: Layout of the points used for PSD calculation.

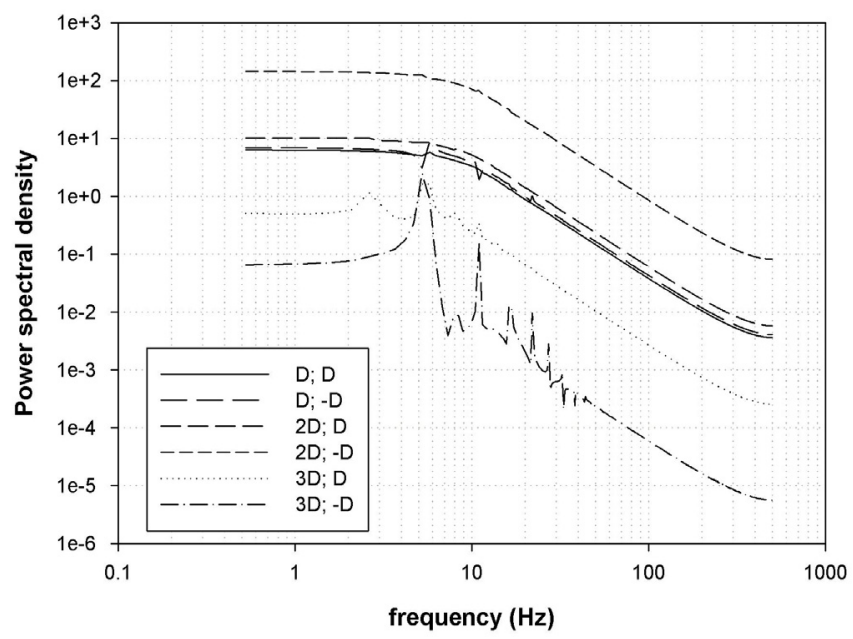

Figure 13: $\quad$ PSD data for the points showed in Fig. 11.

\section{Conclusions}

An efficient computational producer able to analyze the fluid-dynamic behaviour of vertical axis wind turbine was developed. For this purpose an integration between custom routines and a commercial code was performed in order to obtain a good physical model of the phenomenon. The case study presented is related to a Savonius wind rotor. The main features of the flow field developing around the rotor were outlined by means of pressure, relative velocity magnitude contours, relative velocity streamlines and PSD a velocity signal in the rotor wake.

\section{References}

[1] Hansen, M.O.L. Aerodynamics of Wind Turbines, $2^{\text {nd }}$ edition, Earthscan London, Sterling VA, 2008 
[2] S. Montelpare, R. Ricci, V. D’Alessandro, G. Di Giovine. Aerodynamics of a Savonius wind rotor. 4th Symposium on Integrating CFD and Experiments in Aerodynamics, September 14-16, 2009, Ed. by P. Rambaud \& Ph. Planquarat, ISSN 0377-8312, Von Karman Institute for Fluid Dynamics - Rhode-Saint-Genèse, Belgium

[3] V. D’Alessandro, S. Montelpare, R. Ricci, A. Secchiaroli. Unsteady Aerodynamics of a Savonius wind rotor: a new computational approach for the simulation of energy performance. Energy, ISSN 0360-5442, (Accepted. DOI: 10.1016/j.energy.2010.04.021).

[4] R. Ricci, S. Montelpare, G. Borrelli, V. D’Alessandro. Experimental analysis of a Savonius wind rotor for street lighting systems. ASME-ATIUIT Conference on Thermal and Environmental Issues in Energy Systems, May 16-19, 2010, Sorrento, Italy.

[5] Fujisawa, N. Velocity measurements and numerical calculations of flow fields in and around Savonius rotors. J. of Wind Eng. and Ind. Aerod., 59, pp. 39-50, 1996.

[6] Fernando, M.S.U.K., Modi, V.J. A numerical analysis of the unsteady flow past a Savonius wind turbine. J. of Wind Eng. and Ind. Aerod. , 32, Issue 3, pp. 303-327.

[7] Shinohara, T., Ishimatsu, K. Simulation of flow around rotating Savonius rotors. Proc. of the 6th National Symposium on Computational Fluid Dynamics, 691-694 (SEE N94-34731 10-34).

[8] Redchyts, D., Prykhodko, O. Numerical modelling of dynamics and aerodynamics processes of Darrieus and Savonius rotors. Geophysical Research Abstract, 8, 03083, 2006.

[9] Altan, B.D., Atilgan, M. An experimental and numerical study on the improvement of the performance of Savonius wind rotor. En. Conv. and Man., 49, pp., 3425-3432, 2008.

[10] Gupta, R., CFD analysis of a two-bucket Savonius rotor using Fluent package. EWEC 2009, Marseille.

[11] FLUENT users' guide release 6.3.26, Ansys Inc., USA, 2006.

[12] Durbin, P., Near-wall turbulence closure modelling without damping functions. Theoretical and Computational Fluid Dynamics, 3, pp. 1-13, 1991.

[13] Jasak, H., Error analysis and estimation of the finite volume method with applications to fluid flows. PhD thesis - Imperial College of Science, Technology and Medicine, London, UK, 1996.

[14] H.K. Versteeg, W. Malalasekera. An Introduction to Computational Fluid Dynamics, The Finite Volume Method. Longman Scientific \& Technical, 1995. 